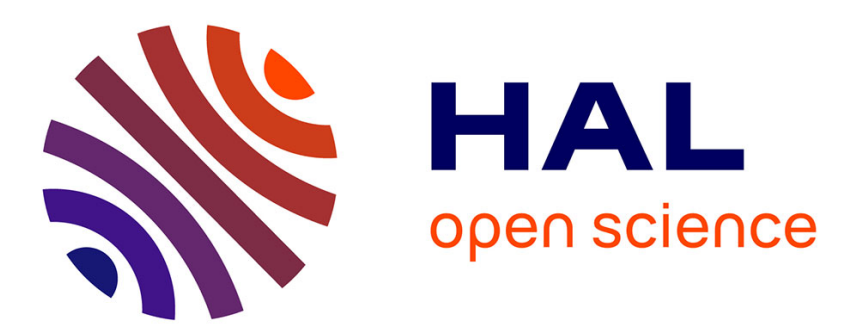

\title{
Time-frequency modeling of shallow water environment: rigid vs. fluid seabed
}

Jun Zhang, Bertrand Gottin, Antonia Papandreou-Suppappola, Cornel Ioana

\section{To cite this version:}

Jun Zhang, Bertrand Gottin, Antonia Papandreou-Suppappola, Cornel Ioana. Time-frequency modeling of shallow water environment: rigid vs. fluid seabed. SSP 2007 - 2007 IEEE workshop on Statistical Signal Processing, Aug 2007, Madison, WI, United States. hal-00326350

\section{HAL Id: hal-00326350 https://hal.science/hal-00326350}

Submitted on 2 Oct 2008

HAL is a multi-disciplinary open access archive for the deposit and dissemination of scientific research documents, whether they are published or not. The documents may come from teaching and research institutions in France or abroad, or from public or private research centers.
L'archive ouverte pluridisciplinaire HAL, est destinée au dépôt et à la diffusion de documents scientifiques de niveau recherche, publiés ou non, émanant des établissements d'enseignement et de recherche français ou étrangers, des laboratoires publics ou privés. 


\title{
TIME-FREQUENCY MODELING OF SHALLOW WATER ENVIRONMENTS: RIGID VS. FLUID SEABED
}

\author{
Jun Zhang ${ }^{\dagger}$, Bertrand Gottin ${ }^{\ddagger}$, Antonia Papandreou-Suppappola ${ }^{\dagger}$ and Cornel Ioana ${ }^{\ddagger}$ \\ ${ }^{\dagger}$ Department of Electrical Engineering, Arizona State University, Tempe, AZ, USA, 85287-7206 \\ ${ }^{\ddagger}$ GIPSA-Lab, INPG/ENSIEG, Grenoble, France
}

\begin{abstract}
When the ocean seabed is considered to be rigid, the ideal waveguide model can be used to model the shallow water environment. However, a more realistic ocean waveguide model treats the ocean floor as a boundary between two different fluid media. In this paper, a frequency-domain characterization of shallow water environments is proposed based on this realistic waveguide model with a fluid boundary. First, the time-frequency characteristics of this model are studied as well as the impact of the environment parameters on the dispersive phenomena. Then a frequency-domain matched filter receiver is designed to obtain time-dispersion diversity once we separate the modes using warping techniques in the time-frequency plane. Simulations demonstrate that the new receiver design improves the bit error rate performance.
\end{abstract}

Index Terms - Time-frequency analysis, dispersive channels, time-frequency mode separation, underwater acoustic communications, time-dispersion diversity

\section{INTRODUCTION}

As a waveform is transmitted in the shallow water environment, it can interact with the ocean bottom and surface, resulting in dense dispersion due to the water medium and timevarying (TV) changes of the ocean environment. Specifically, the shallow water acoustic environment is a linear TV dispersive system that shifts different frequencies by different amounts in time [1].

The dispersive effect introduced by shallow water environments can severely limit the performance of underwater acoustic applications such as sonar and communications. It can be shown that the propagation characteristics of an environment can provide us with a specific nonlinear function that defines the dispersion in this environment. Thus, the corresponding signal representation based on a normal mode model can be applied to exploit the potential diversity suggested by the model when the acoustic receiver is appropriately designed to match this nonlinear function.

*This work was supported by the NSF CAREER Award CCR-0134002 and the Department of Defense Grant No. AFOSR FA9550-05-1-0443.
In [1], a shallow water characterization was considered that matched dispersive signal transformations on the transmitted waveforms and was used successfully for shallow water communications to obtain time-dispersion diversity. However, this characterization was only applicable to signals with very high bandwidth as it assumed that the transmitted waveform was an impulse.

In [2], we presented a generalized characterization that was applicable to a larger class of signals by using the normalmodel in [3] assuming perfect waveguide conditions (homogeneous fluid layer with a soft top and rigid bottom). In this paper, we extend our characterization when these conditions are not perfect by following the waveguide model with pressure-release surface and fluid boundaries in [4]. Using the new model, we propose a new time-frequency receiver design which operates on individual modes of the multi-component received signal instead of on the whole signal. This upgraded receiver design of an underwater communication system is expected to achieve better bit error rate (BER) performance for time-dispersion diversity than the one obtained in [2].

The paper is organized as follows. In Section 2, we formulate the Pekeris model for shallow water environments. In Section 3, we present the time-frequency (TF) characteristics of this model, and we investigate the impact of the environment parameters on the dispersion effect. In Section 4, we use a TF mode separation technique to separate each component of the received signal and then design a new receiver structure with a corresponding optimal detector. In Section 5, numerical results of BER performance illustrate our improved performance in terms of diversity order.

\section{MODELING FOR SHALLOW WATER ENVIRONMENT: PEKERIS WAVEGUIDE}

The normal-mode model treats the ocean as a waveguide with plane, parallel boundaries, representing the acoustic field in the ocean medium as a sum of normal modes. We first discuss the normal-mode model for shallow water environments with pressure-release surface and a fluid seabed following [4, 5, 3]. A simple waveguide model of the ocean is shown in Fig. 1 using the coordinate system $(r, z)$, where Medium I, II and 


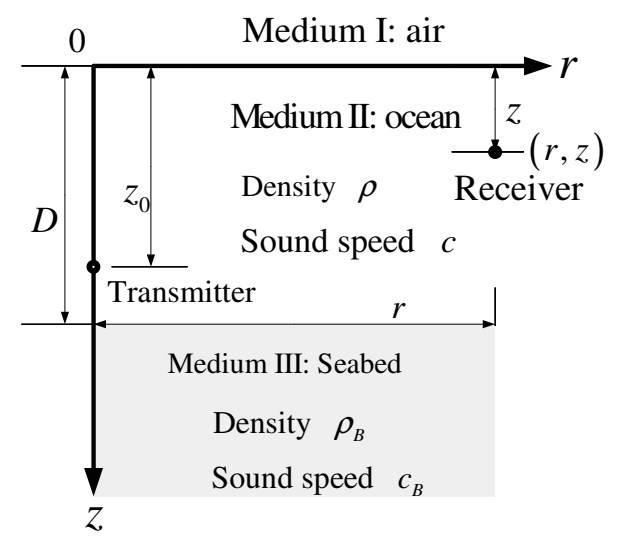

Fig. 1. Waveguide model with point source in Medium II at $r=0, z=z_{0}, D \mathrm{~m}$ deep. This corresponds to a pressure release surface with a fluid seabed environment [4].

III correspond to air, ocean water and seabed, respectively. An omnidirectional point source with signal spectrum $X(f)$ is located in Medium II at $r=0$ and $z=z_{0}$. The ocean is $D \mathrm{~m}$ deep. We consider the sound speed in Medium II as a constant $c \mathrm{~m} / \mathrm{s}$ and density $\rho \mathrm{kg} / \mathrm{m}^{3}$, and in Medium III as a constant $c_{B} \mathrm{~m} / \mathrm{s}$ and density $\rho_{B} \mathrm{~kg} / \mathrm{m}^{3}$.

The ocean surface (at $z=0$ ) is modeled realistically as an ideal pressure release boundary and the ocean bottom (at $z=D$ ) is modeled as a boundary between two different fluid media. The normal mode model is given by the solution of this ocean waveguide problem, which is determined by the environment parameters and satisfies all boundary conditions, including the boundary condition at the source.

After a detailed derivation in [4] and [5], the received signal spectrum excited by $X(f)$ at location $(r, z)$ is given by the Pekeris waveguide model:

$$
Y_{\text {Pekeris }}(f)=X(f) \sum_{n=0}^{N_{p}-1} C_{n}(f) \Theta_{n}(f) .
$$

Without the assumption of ideal waveguide condition as in [2], the $n$th mode is characterized by

$$
\Theta_{n}(f)=\sqrt{\frac{1}{k_{n}(f) r}} e^{-j k_{n}(f) r},
$$

where

$k_{n}(f)=\frac{2 \pi}{c}\left(f^{2}-\frac{n^{2} c^{2}}{4 D^{2}}\left(1+\frac{\rho_{B} c}{\rho D} \frac{1}{2 \pi f \sqrt{1-\frac{c^{2}}{c_{B}^{2}}}}\right)^{-2}\right)^{\frac{1}{2}}$

is the wave number of the $n$th mode and $N_{p}$ is the largest mode number. The parameter

$$
C_{n}(f)=A_{n}^{2}(f) \sin \left(k_{z n}(f) z_{0}\right) \sin \left(k_{z n}(f) z\right)
$$

is a function of frequency where

$$
\begin{aligned}
& A_{n}(f)=\sqrt{2}\left[\frac{1}{\rho}\left(D-\frac{\sin \left(2 k_{z n}(f) D\right)}{2 k_{z n}(f)}\right)\right. \\
& \left.-\frac{\rho}{\rho_{B}^{2}} \frac{\tan \left(k_{z n}(f) D\right) \sin ^{2}\left(k_{z n}(f) D\right)}{k_{z n}(f)}\right]^{-\frac{1}{2}} \\
& \text { and } k_{z n}(f) \approx \frac{n \pi}{D}\left(1+\frac{\rho_{B} c}{\rho D} \frac{1}{2 \pi f \sqrt{1-\frac{c^{2}}{c_{B}^{2}}}}\right)^{-1} \text {. }
\end{aligned}
$$

\section{TIME-FREQUENCY CHARACTERISTICS OF SHALLOW WATER ENVIRONMENT MODEL}

The TF characteristic of the acoustic signal is determined by the modal group velocity $(\mathrm{MGV}) g_{n}(f)$, due to the propagating delay of the frequency $f_{0}$ in the $n$th mode is determined by $\tau_{n}\left(f_{0}\right)=\frac{r}{g_{n}\left(f_{0}\right)}$. The modal group velocity is shown in Fig. 2. From Fig. 2, we notice that, for the waveguide model with fluid seabed, the MGV approaches $c$ when the frequency approaches infinity, and approaches $c_{B}$ when the frequency approaches the cutoff frequency of this mode.

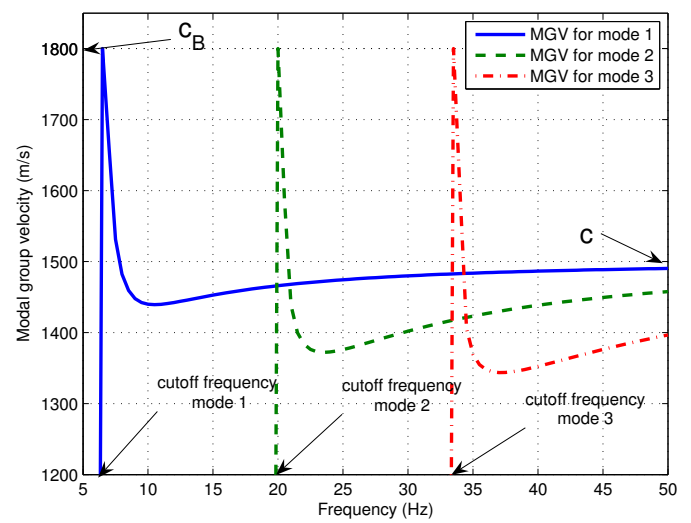

Fig. 2. Modal group velocity (MGV) function.

We analyzed further the TF characteristics of the received signal using the spectrogram TF representation (TFR) and investigated the effect of changing the parameters of the environment. Fig. 3(a) represents the spectrogram for three modes with environment parameters: $D=100 \mathrm{~m}$ and $r=15 \mathrm{~km}$. Fig. 3(b) uses the same set of parameters except that $r=30$ km. Fig. 3 shows each mode as a dispersive curve in the TF plane. We note that the curves stop at the cutoff frequency with no asymptotical behavior to the cutoff frequency. Fig. 3 shows that longer range between the receiver and transmitter causes more dispersion in the received signal. Fig. 4 shows that with larger ocean depth, the modal cutoff frequency is lower, and with a smaller depth, the dispersive effect is more visible at higher frequencies. 

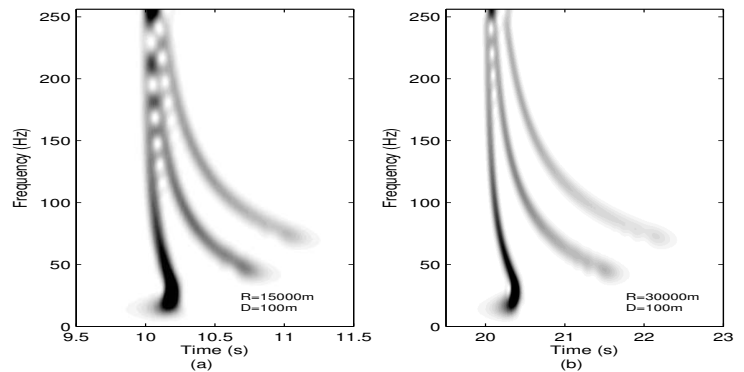

Fig. 3. Spectrograms with environment parameters (a) $r=15$ $\mathrm{km}, D=100 \mathrm{~m}$, and (b) $r=30 \mathrm{~km}, D=100 \mathrm{~m}$.
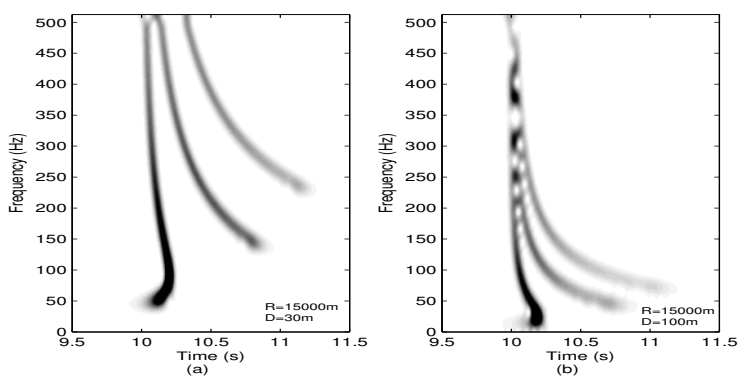

Fig. 4. Spectrograms with environment parameters (a) $r=15$ $\mathrm{km}, D=30 \mathrm{~m}$, and (b) $r=15 \mathrm{~km}, D=100 \mathrm{~m}$.

\section{SYSTEM DESIGN FOR COMMUNICATION DIVERSITY}

According to the Pekeris waveguide in (1), the received signal spectrum is the superposition of multiple normal modes. Considering each mode as a communication subchannel, the time-dispersive channel can be interpreted as a superposition of subchannels, and using their TF characteristics, we can separate each subchannel and design the corresponding receiver to exploit time-dispersion diversity.

\subsection{Separation of normal modes}

Fig. 3 illustrates that each mode of the received signal appears as a dispersive curve in the TF plane, which makes the separation of the modes possible. In this paper, we use a two-step TF mode separation technique based on warpings to separate the modes. The two steps are described as follows.

During the first step, we design $N_{p}-1$ instantaneous frequency (IF) curve separators in the TF plane, represented as $e_{n}(t), n=0, \cdots, N_{p}-2$. An IF curve separator is a curve situated between two successive modes in the TF plane as shown in Fig. 5(a) for the example in Fig. 3. In this problem, all environmental parameters are assumed to be known, so that the TF structures of the received signal are also known
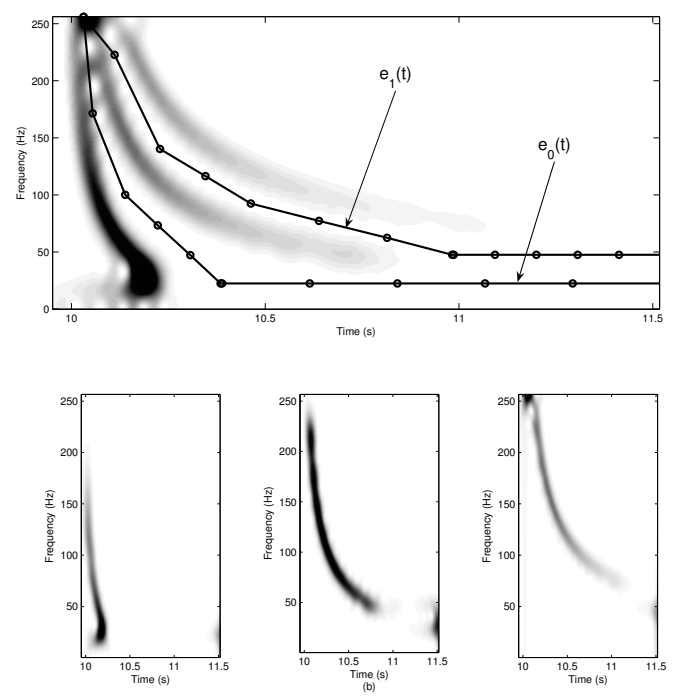

Fig. 5. (a) Spectrogram of received signal with the IF curve separators, (b) separated TF modes.

using the model in (1). Knowing the TF structures, we appropriately set $M$ TF points $\left(t_{i}, f_{i}\right), i=1,2, \ldots, M$ in the middle of the space between two successive modes. The $M$ points between the $n$th mode and the $(n+1)$ th mode will constitute the IF curve separator $e_{n}(t)$. In the next step, the IF curves will be processed with the received signal. Thus, the separation lines must have the same duration as the received signal. To ensure that, all the IF curves begin at the starting point of the TF structure and are extended with a straight horizontal line until the end of the TF window.

During the second step, we process the different modes in the TF plane using a warping technique $[6,7]$ based on the use of the local harmonic convolution operator [6]. Assume that the received noisy signal in (1) is $r(t)$ with Fourier transform (FT) $R(f)=Y_{\text {Pekeris }}(f)+W(f)$, where $W(f)$ is additive white Gaussian noise. We then obtain the warping functions from the IF curve separators as $\zeta_{n}(t)=\frac{1}{f_{c}} \int_{-\infty}^{t} e_{n}(u) d u$, where $f_{c}>0$, and compute the corresponding generalized FT of $r(t)$ as [8]

$$
\mathcal{M}_{r}(\lambda)=\int_{\wp} r(t) \frac{d \zeta_{n}(t)}{d t} e^{-j 2 \pi \lambda \zeta_{n}(t)} d t,
$$

where $\wp$ contains the values of $t$ in the domain of the warping function $\zeta_{n}(t)$, and $\lambda$ is a real and unitless parameter. To obtain the first mode, we compute the inverse generalized FT over the range $\left(-\infty, f_{c} / f_{r}\right]$ as follows:

$$
r_{0}(t)=\int_{-\infty}^{f_{c} / f_{r}} \mathcal{M}_{r}(\lambda) e^{j 2 \pi \lambda \zeta_{0}(t)} d \lambda,
$$

where $f_{r}$ is a normalization frequency. Then we subtract the 
first mode from the received signal, i.e, $\hat{r}(t)=r(t)-r_{0}(t)$, and the remaining modes are contained in $\hat{r}(t)$.

To obtain the seconde mode, we apply the above procedure to $\hat{r}(t)$ using the IF curve separator $e_{1}(t)$. Repeating the above procedure for each IF curve separator, we can then separate each mode from the received signal.

For the example in Fig. 5, we use the mode separation technique on the received signal excited by the waveform $X(f)=\sqrt{f}$ for $N_{P}=3$ modes. The IF curve separators are shown in Fig. 5(a) and the separated components are shown in Fig. 5(b).

\subsection{Receiver structure}

Under the Pekeris waveguide assumption, the model in (1) can be seen as an inherent frequency domain transfer function. However, due to the distortion and signal propagation uncertainty in realistic shallow water environments, a more suitable model of random signal embedded in random noise consists of

$$
R(f)=Y(f)+W(f)=X(f) \sum_{n=0}^{N_{p}-1} D_{n} C_{n}(f) \Theta_{n}(f)+W(f),
$$

where $R(f)$ is the spectrum of the received signal, $D_{n}$ is a random process incorporating amplitude and phase variability of the source, and $W(f)$ is additive white Gaussian noise with variance $\sigma_{W}^{2}$.

We propose a filter bank receiver scheme based on this signal model which can exploit the frequency domain dispersion diversity existing in the normal modes. The received signal spectrum $R(f)$ in (5) is first processed as described in Section 4.1 to separate each mode, and then the outputs are combined in the minimum-probability-of-error sense to obtain the transmitted information symbol $b$. This receiver structure is illustrated in Fig. 6.

We design the transmission waveform as $X(f)=\sqrt{f}$ for $f \geq f_{0}$ where $f_{0}$ is the cutoff frequency of the first mode, and then we employ the corresponding frequency domain matched filter to each separated mode component of the received signal. The $n$th mode component obtained from the separation processing is expressed as

$$
R_{n}(f) \approx b X(f) D_{n} C_{n}(f) \Theta_{n}(f)+W_{n}(f),
$$

where $W_{n}(f)$ is non-white Gaussian noise, with variance $\sigma_{W_{n}}^{2}$, that resulted from $W(f)$ when the $n$th mode is separated.

We first define the receiver matched waveform $U_{n}(f)=$ $X(f) C_{n}(f) \Theta_{n}(f)$ for the $n$th mode. Then, as each mode is separated, we can perform individual matched filtering on the $n_{0}$ th mode by $D_{n_{0}} U_{n_{0}}(f)$; this can avoid interference with the other modes. The output of the $n_{0}$ th matched filter can be

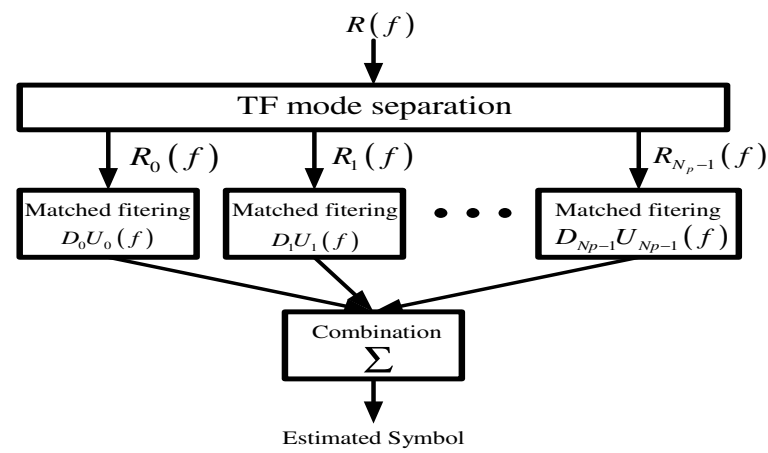

Fig. 6. Receiver structure scheme.

expressed as

$$
Z_{n_{0}}=\left\langle R_{n_{0}}(f), D_{n_{0}} U_{n_{0}}(f)\right\rangle=\int_{f} R_{n_{0}}(f) D_{n_{0}}^{*} U_{n_{0}}^{*}(f) d f .
$$

Using the expression of $R_{n}(f)$ in (6), we can rewrite (7) as

$Z_{n_{0}} \approx b\left|D_{n_{0}}\right|^{2} \int_{f} U_{n_{0}}(f) U_{n_{0}}^{*}(f) d f+\int_{f} W_{n_{0}}(f) D_{n_{0}}^{*} U_{n_{0}}^{*}(f) d f$.

As the output of this filter bank is given by the concatenation $\left[\begin{array}{llll}Z_{0} & \ldots & Z_{N_{p}-1}\end{array}\right]$, the combination in the minimumprobability-of-error sense is defined as the arithmetical mean of the filtering outputs. Thus, the estimation of the transmitted information symbol can be expressed as

$$
\hat{b}=\Re\left(\sum_{n=0}^{N_{p}-1} Z_{n}\right) .
$$

where $\Re(\cdot)$ is the operator that takes the real part. Assuming an antipodal symbol transmission, i.e, $b=+1$ or $b=-1$, and that the probabilities of transmitting +1 and -1 are equal, the minimum error probability detector can be expressed as:

$$
\begin{aligned}
& \text { decide } b=+1 \text { if } \hat{b}>0, \\
& \text { decide } b=-1 \text { if } \hat{b}<0 .
\end{aligned}
$$

\section{PERFORMANCE ANALYSIS}

As the modes are separated, each mode can be considered as a subchannel, and the aforementioned matched filter receiver can avoid cross-correlation interferences between different modes of the received signal. Hence, this matched filter receiver can exploit the potential diversity existing among the different modes. In this section, we first derive the theoretical probability of error for the aforementioned receiver, and then we obtain the BER results from numerical simulations. We also compare the performance of this receiver with the performance of a receiver that processes the complete received signal. 
Let us consider the matrix of frequency domain mode autocorrelations defined as $\mathbf{A}=\operatorname{diag}\left(A_{0}, A_{1}, \ldots, A_{N_{P}-1}\right)$ where

$$
A_{i}=\int_{f} U_{i}(f) U_{i}^{*}(f) d f, \quad i=0, \ldots, N_{P}-1
$$

and the matrix of modal noise variance $\sigma=\operatorname{diag}\left(\sigma_{W_{i}}^{2}\right)$ for $i=0, \ldots, N_{P}-1$. Defining $\sum=\sigma^{-1} \sqrt{\mathbf{A}} Q \sqrt{\mathbf{A}}^{\dagger}$ and $\mathbf{m}=\sqrt{\sigma^{-1} \mathbf{A}} \mathrm{E}[\mathbf{D}]$, where $\mathrm{E}[\cdot]$ is the expectation operator and $Q$ is the covariance matrix of the coefficients vector $\mathbf{D}=\left[D_{0}, D_{1}, \ldots, D_{N_{P}-1}\right]^{\mathcal{T}}$, we can derive the theoretical BER as the probability of error given by [2]:

$$
P_{\text {err }}=\frac{1}{\pi} \int_{0}^{\frac{\pi}{2}}\left[\operatorname{det} \frac{\sum}{\sin ^{2} \theta}+\mathbf{I}\right]^{-1} e^{-\mathbf{m}^{\dagger}\left(\sum+\sin ^{2} \theta \mathbf{I}\right)^{-1} \mathbf{m}} d \theta,
$$

where we denote $\mathcal{T}$ and $\dagger$, respectively, as the transpose and conjugate transpose operations. The BER obtained from the simulation and the theoretical expression are shown in Fig. 7, using the environment parameters $D=100 \mathrm{~m}, r=15 \mathrm{~km}$, $c=1500 \mathrm{~m} / \mathrm{s}, c_{B}=1800 \mathrm{~m} / \mathrm{s}, \rho=1000 \mathrm{~kg} / \mathrm{m}^{3}$ and $\rho_{B}=$ $1800 \mathrm{~kg} / \mathrm{m}^{3}$ (as in Figures 3 and 5). The results show the $\mathrm{BER}$ as a function of the signal-to-noise ratio (SNR) for the transmission frequency band $5-250 \mathrm{~Hz}$.

We notice from Fig. 7 that the simulation results are worse than the theoretical results. This is because the mode separation technique caused loss of information, especially at higher frequencies, which leads to less accurate matched filtering. However, if we assume no information is lost in the procedure of separation, i.e, the separation can generate the exact modes, then the numerical simulation results are close to the theoretical results, as expected.

We also compared the BER performance of the new receiver with the performance obtained in [2] where the matched filtering was applied to the whole multi-component received signal. Fig. 7 shows that the new receiver performed better according to the numerical simulation results.

\section{CONCLUSION}

We developed a frequency domain characterization of realistic shallow water environments and analyzed its dispersive characteristics. Following this model, we developed the appropriate receiver design based on using a warping technique for modal separation. This receiver structure aims to exploit the diversity existing in the system. Simulation results show that BER performance and diversity order were improved by the aforementioned system design.

\section{REFERENCES}

[1] Y. Jiang and A. Papandreou-Suppappola,"Discrete timefrequency characterizations of dispersive time-varying

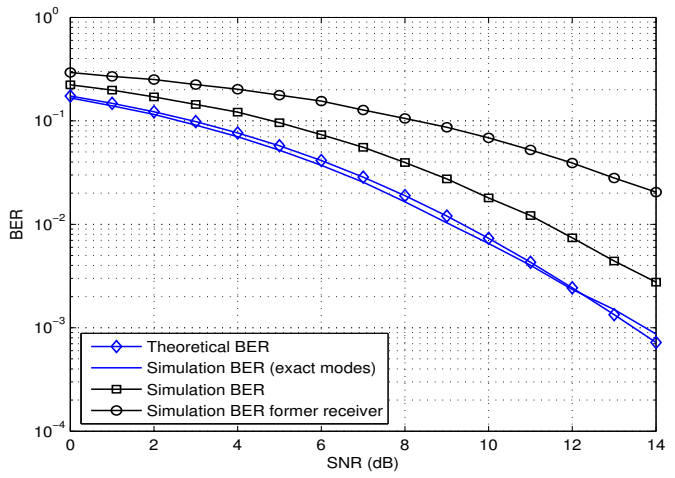

Fig. 7. Theoretical BER performance of the new receiver (diamonds), BER performance of the new receiver obtained by numerical simulation (squares), BER performance of the new receiver obtained by numerical simulation using exact modes (line) and BER performance of the former receiver (circles).

systems," IEEE Trans. Signal Processing, vol. 55, pp. 2066-2076, May 2007.

[2] J. Zhang and A. Papandreou-Suppappola, "Timefrequency based waveform and receiver design for shallow water communications," in Proc. IEEE Int. Conf. on Acoust., Speech, Signal Processing, vol. 3, pp. 11491152, April 2007.

[3] L. J. Ziomek, Fundamentals of Acoustic Field Theory and Space-Time Signal Processing, CRC Press, 1999.

[4] G. V. Frisk, Ocean and Seabed Acoustics, Prentice-Hall, 1994.

[5] G. B. Deane, "Internal friction and boundary conditions in lossy fluid seabeds," The journal of the Acoustical Society of America, vol. 101, no. 1, pp. 233-240, January 1997.

[6] A. Jarrot, C. Ioana, C. Gervaise and A. Quinquis, "A class of linear time-varying filters based on non-unitary timewarping operators. Part I: principles and examples," IEEE Trans. Signal Processing, submitted, 2006.

[7] A. Jarrot, C. Ioana, C. Gervaise and A. Quinquis, "A timefrequency characterization framework for signals issued from underwater dispersive environments," in Proc. IEEE Int. Conf. on Acoust., Speech, Signal Processing, vol. 3, pp. 1145-1148, April 2007.

[8] A. Papandreou-Suppappola, "Time-Varying Processing: Tutorial on Principles and Practice,"in Applications in Time-Frequency Signal Processing, (A. PapandreouSuppappola, ed.), Florida: CRC Press, pp. 1-84, 2002. 